\title{
Outpatient antibiotic prescribing behavior and their psychosocial predictors among early-career clinicians in Delhi, India
}

\author{
Saurav Basu ${ }^{1}$, Sahadev Santra ${ }^{1}$, Nidhi Bhatnagar ${ }^{1}$, and Anish Laul ${ }^{1}$ \\ ${ }^{1}$ Maulana Azad Medical College
}

April 27, 2020

\begin{abstract}
Background: Psychosocial factors are significant drivers of inappropriate antibiotic prescription leading to antibiotic resistance (ABR). Objective: To ascertain the psychosocial predictors of outpatient antibiotic prescribing behavior among early-career clinicians in India. Methods: We enrolled 200 early-career clinicians, including 100 medical interns and 100 junior residents (postgraduate student doctors) in six clinical departments, and collected data using a self-administered questionnaire. Response options were coded on five-item Likert scales. Results: Antimicrobial resistance (AMR) was viewed as a significant public health problem by most (95\%) participants. Presumptive antibiotic prescribing was reported by $84 \%$ of participants, although the participant attitude indicated a slight disinclination against the presumptive use of antibiotics (Mean $=2.8, \mathrm{SD}=0.72$ ). The majority $(52.5 \%)$ of the participants' perceived social pressure frequently influenced their decision to prescribe antibiotics to their outpatients, which was most commonly attributed to patient expectation for antibiotics and from observing the antibiotic prescribing behavior of their peers and colleagues. The perception of increased social pressure stipulating antibiotic prescribing negatively correlated with the participant's intention to reduce antibiotic use in outpatients $(\mathrm{r}=-0.124$, p $<0.001)$. Social pressure was reported to be higher when treating adult patients reporting diarrheal symptoms, and children having cough. Conclusions: Outpatient antibiotic prescribing practices among early-career clinicians working in the government health sector in India are mediated by considerable social pressure despite their perceived intention for reducing antibiotic use.
\end{abstract}

\section{INTRODUCTION}

The emergence of antibiotic resistance (ABR) as a major global public health challenge is an outcome of the increasing use and misuse of antibiotics, which reduces the effectiveness of these lifesaving drugs against disease-causing microbes (1). Inappropriate antibiotic prescription by clinicians, especially in outpatient settings which constitute the most common sites of antibiotic dispensing, contributes enormously to the problem of antibiotic resistance $(2,3)$. The failure to prevent ABR results in millions of avoidable deaths each year, which in the absence of effective intervention, could scale 10 million deaths by 2050 (4). There is also growing recognition of the negative impact of antibiotic exposure especially during infancy and early life on the microbiome in the human body which predisposes to chronic diseases in adolescence and adulthood (5).

Unwarranted antibiotic prescriptions in outpatient settings can account for nearly one in two inappropriate prescriptions, even in developed countries like the USA (6). In resource-constrained environments, the risk of unnecessary antibiotic prescription is further accentuated due to the widespread practice of presumptive or empirical therapy, when antibiotics, especially of broad-spectrum type, are prescribed without considering results of appropriate microbiological investigations (7). Furthermore, social and ethical dilemmas also regulate the physician's antibiotic prescribing antibiotics, particularly when combined with a lack of effective regulation and oversight $(8,9)$. The identification of psychosocial determinants of clinicians' outpatient antibiotic prescribing practices in India is pivotal due to its applicability in the formulation of appropriate public health interventions for combating ABR. 
We, therefore, conducted this study to ascertain the psychosocial predictors of outpatient antibiotic prescribing behavior among early career clinicians in India.

\section{METHODS}

Study site, participants and setting

We conducted a cross-sectional study at a government medical college and affiliated tertiary care hospital in Delhi, India, and enrolled early-career clinicians, including medical interns and junior resident (postgraduate student) doctors from the clinical departments.. We selected only those interns who had already completed internship training both in the medicine (hospital outpatient) and the community medicine department (outreach primary care settings), which ensured adequate experience in the dispensing of antibiotics. Data were collected from September-December' 2019

No formal outpatient antibiotic stewardship training program as part of continuing medication education or training was available for the interns and postgraduate students of the institute. Diagnostics were available free of cost at the hospital laboratory, but there were long waiting-queues due to high patient-load. Furthermore, outpatients of low socioeconomic status were unwilling to spend out of pocket for routine diagnostics. Under these circumstances, considering the effective high laboratory turnabout time in obtaining outpatient reports, presumptive antibiotic prescribing is a ubiquitous practice in the study settings

Sampling : the study participants were enrolled through the consecutive sampling method in six clinical departments and were contacted after their morning or afternoon outpatient clinic hours.

Study instruments

We used a self-administered questionnaire for data collection, which included questions adapted from the previously validated instrument by Liu et al. after obtaining permission from the authors. ${ }^{[10]}$ The questionnaire measured attitudes, subjective norms, behavioral control, behavioral intentions, and awareness, and perspectives on dysbiosis in the prescribers. All the responses were recorded on a five-item Likert scale (Annexure 1).

1. Attitudes (5 items): were defined as the perceived attitude of the clinicians' towards the practice of presumptive antibiotic therapy in terms of the usefulness, appropriateness, and responsibleness with higher scores indicating a more favorable view.

2. Subjective norms (13 items): assessed social pressure perceived by the physician in clinical situations that required antibiotic prescribing related decision-making. The participants rated the extent of perceived pressure arising from patient expectations, peer prescribing behavior, peer expectations, social and familial obligation, and as per the patient clinico-demographic profile.

3. Perceived behavioral control (5 items): it measured the confidence and ease of the physician in deciding whether to prescribe antibiotics to their patients or not.

4. Behavioral intentions ( 7 items): it measures if the physician desired to change their frequency of antibiotic use, either by increasing or reducing prescription while adhering to the recommended local antibiotic stewardship guidelines.

Statistical analysis: We analyzed the data with IBM SPSS Version 25. Likert item responses were reverse coded before scoring for questions relating to perceived behavioral control and behavioral intention. Results were expressed in frequency and proportions for categorical variables, and the median and mean value was reported for the Likert scale items. Continuous outcomes were expressed as mean and standard deviation. A p-value $<0.05$ was considered statistically significant.

\section{RESULTS}

We enrolled a total of $143(57 \%)$ men and $86(43 \%)$ women participants, comprising 100 medical interns and 100 resident doctors undergoing postgraduate training. 
The Cronbach's Alpha of the subscales including attitude, subjective norms, perceived behavioral control and behavioral intention was $0.78,0.82,0.63$ and 0.61 respectively suggestive of acceptable reliability.

Perspectives on antimicrobial resistance (AMR) and outpatient antibiotic prescribing (OAP) : AMR was perceived as a significant public health problem by most (95\%) participants, and the mean (SD) score of the ABR threat rated on a 5 point continuous rating scale was $3.89(0.88)$. The role of OAP contributing to the problem of ABR was viewed as 'somewhat likely' by $14(7 \%)$, likely by 96 (48\%) and very likely $83(41.5 \%)$ participants. The presumptive use of antibiotics in outpatients was practiced 'sometimes' by $119(59.6 \%)$, 'often' by 46 (23\%), 'always' by 3 (1.5\%), and 'rarely/never' by 32 (16\%) participants. Most (80.5\%) participants also perceived their antibiotic prescribing behavior was consistent with autonomous decision making and expressed their intention to further reduce antibiotic use (91.5\%) in their outpatients (Table 1).

Attitude and practices towards presumptive antibiotic prescribing in outpatients : The attitude of the participated indicated a slight disinclination against the presumptive use of antibiotics (Mean $=2.8, \mathrm{SD}=$ 0.72). The majority $(52.5 \%)$ of the participants' agreed that social pressure could influence their decision to prescribe antibiotics for their outpatients. The maximum social pressure was attributed to patient expectations for antibiotics and observing antibiotic prescribing behavior in their peers and colleagues (Table 2 ). When considering the clinico-demographic profile of patients, social pressure was reported to be maximum when treating adult patients reporting symptoms of diarrhea, and children having cough (Table 3). However, the participants were also positively inclined towards reducing their antibiotic use in outpatients $($ Mean $=4.2,0.68)$, while adhering to the recommended antibiotic prescribing guidelines (Mean $=4.2, \mathrm{SD}$ $=0.72$ ). Increasing social pressure negatively correlated with the participant's intention to reduce antibiotic prescription for outpatients $(\mathrm{r}=-0.124, \mathrm{p}<0.001)$.

\section{DISCUSSION}

Psychosocial factors are major drivers of inappropriate antibiotic prescription (2). The present study observed significant self-reported social pressures and social obligations influencing outpatient antibiotic prescribing practices among early career clinicians in Delhi, India. However, nearly four in five participants perceived themselves having adequate behavioral control needed for adhering to rational antibiotic prescription guidelines and in reducing undesirable antibiotic use. The results of our study, in comparison with findings from a large cross-sectional study among primary care providers in China, show similar levels of social pressure but a comparatively stronger perception of self-control in regulating antibiotic prescription in their outpatients. ${ }^{[10]}$ Nevertheless, it is well-established that physician intention is a weak predictor of antibiotic prescribing behavior in scenarios where the physician is unable to generate a reliable diagnosis as during presumptive prescribing (11). Concern over the development of complications in patients, especially if socioeconomically vulnerable, can also decisively influence antibiotic prescribing decisions $(8,12)$.

We found social pressure in prescribing antibiotics varied with the presenting symptoms and the age-profile of the patient. Clinicians perceived higher social pressure when treating diarrheal conditions in adults, similar to the finding from a previous study conducted among primary-care physicians in Delhi (13). We also observed higher social pressure in clinicians was associated with reduced self-efficacy for reducing antibiotic usage in outpatients, a result in agreement with a Chinese study (10).

In conclusion, outpatient antibiotic prescribing practices in early-career clinicians working in the government health sector in India is mediated by considerable social pressure despite identifying the threat of ABR and reporting strong behavioral intentions towards reducing outpatient antibiotic use. Future studies need to identify the effect of behavioral interventions among clinicians for overcoming social pressures and strengthening outpatient antibiotic stewardship for the promotion of rational prescribing practices.

Limitations : First, the study was conducted in a government hospital setting, which may attenuate social and financial obligations involved in antibiotic prescription to patients, compared to the private medical sector that caters to the majority of the population (14). Second, the participants were enrolled from a single site in Delhi, and the findings cannot be extrapolated to varied regional settings. Third, we did not 
corroborate the subjective measure of physician self-reported antibiotic prescribing behavior by linking it with their prescription data. Fourth, the correct knowledge of antibiotic stewardship may influence the clinician's prescribing decision (15), but this was not assessed in this study. Fifth, behavioral intention to reduce antibiotic prescription can be over-reported by the participants due to the social desirability bias.

Funding : Nil

\section{The authors declare they have no competing interests}

Ethical considerations : The study was approved with exemption from full review by the Institutional Ethics Committee, Maulana Azad Medical College \& Associated Hospitals, New Delhi (F.1/IEC/MAMC/(68/03/2019/No/68 Written and informed consent was obtained from all the study participants.

Author contributions : SB and NB designed and conceptualized the study, SS and AL were involved in data collection and management, SB and SS contributed to the literature review, SB conducted the statistical analysis and wrote the first draft of the manuscript. All authors provided intellectual content and were involved in critical editing and revision of the manuscript.

\section{REFERENCES}

1. WHO. Global action plan on antimicrobial resistance. Geneva: World Health Organization; 2015.

2. King LM, Fleming-Dutra KE, Hicks LA. Advances in optimizing the prescription of antibiotics in outpatient settings. BMJ. 2018;363:k3047.

3. Laxminarayan R, Duse A, Wattal C, Zaidi AK, Wertheim HF, Sumpradit N, et al. Antibiotic resistancethe need for global solutions. Lancet Infect Dis. 2013;13(12):1057-98.

4. O' Neil J. Review on antimicrobial resistance antimicrobial resistance: tackling a crisis for the health and wealth of nations. London: Review on Antimicrobial Resistance; 2014.

5. Carding S, Verbeke K, Vipond DT, Corfe BM, Owen LJ. Dysbiosis of the gut microbiota in disease. Microb Ecol Health Dis. 2015;26:26191.

6. Shapiro DJ, Hicks LA, Pavia AT, Hersh AL. Antibiotic prescribing for adults in ambulatory care in the USA, 2007-09. J Antimicrob Chemother. 2014;69(1):234-40.

7. Leekha S, Terrell CL, Edson RS. General principles of antimicrobial therapy. Mayo Clin Proc. 2011;86(2):156167.

8. Basu S, Garg S. Antibiotic prescribing behavior among physicians: ethical challenges in resource-poor settings. J Med Ethics Hist Med. 2018;11:5.

9. Mao W, Vu H, Xie Z, Chen W, Tang S. Systematic review on irrational use of medicines in China and Vietnam. PLoS One. 2015;10(3):e0117710.

10. Liu C, Liu C, Wang D, Deng Z, Tang Y, Zhang X. Determinants of antibiotic prescribing behaviors of primary care physicians in Hubei of China: a structural equation model based on the theory of planned behavior. Antimicrob Resist Infect Control. 2019;8:23.

11. Lambert BL, Salmon JW, Stubbings J, Gilomen-Study G, Valuck RJ, Kezlarian K. Factors associated with antibiotic prescribing in a managed care setting: an exploratory investigation. Soc Sci Med. 1997;45(12):1767-79.

12. Sanchez GV, Roberts RM, Albert AP, Johnson DD, Hicks LA. Effects of knowledge, attitudes, and practices of primary care providers on antibiotic selection, United States. Emerg Infect Dis 2014;20:2041-7.

13. Kotwani A, Wattal C, Katewa S, Joshic PC, Holloway K. Factors influencing primary care physicians to prescribe antibiotics in Delhi India. Fam Pract. 2010;27(6):684-90 
14. Farooqui HH, Mehta A, Selvaraj S. Outpatient antibiotic prescription rate and pattern in the private sector in India: Evidence from medical audit data. PLoS One. 2019;14(11):e0224848.

15. Garcell HG, Arias AV, Sandoval CP, Valle Gamboa ME, Sado AB, Alfonso Serrano RN, et al. Impact of a focused antimicrobial stewardship program in adherence to antibiotic prophylaxis and antimicrobial consumption in appendectomies. J Infect Public Health 2017;10:415-20.

Table 1. Attitudes, perceptions and practices related to outpatient antibiotic prescribing among clinicians in Delhi $(\mathrm{N}=200)$

\begin{tabular}{llll}
\hline Measurement domain & Mean (SD) & Median & (\%) of Scores > $3^{+}$ \\
\hline Attitude & $2.8(0.72)$ & 3 & 32.5 \\
Perceived social pressure & $3.2(0.5)$ & 3.1 & 52.5 \\
Perceived behavioral control & $3.53(0.49)$ & 3.6 & 80.5 \\
Behavioral intention to reduce antibiotic prescription & $3.8(0.46)$ & 3.7 & 91.5 \\
\hline
\end{tabular}

+ Maximum score $=5$

Table 2. Perceived social pressure in prescribing antibiotics to outpatients among clinicians in Delhi $(\mathrm{N}=$ 200)

\begin{tabular}{lll}
\hline Psychosocial factor & Median perceived social pressure & $(\%)$ of Scores $>3^{+}$ \\
\hline Patient expectations & 4 & 59.5 \\
Peer behavior & 4 & 64.5 \\
Senior peer behavior & 3 & 39 \\
Perceived workplace expectation & 3 & 29 \\
Social obligation towards friends & 3 & 22 \\
Social obligation promoting inappropriate antibiotic prescribing & 3 & 21 \\
\hline
\end{tabular}

+ Reported as 'often' or 'always' (maximum score $=5$ )

Table 3. Perceived social pressure in prescribing antibiotics to outpatients influenced by the patient clinicodemographic profile $(\mathrm{N}=200)$

\begin{tabular}{lll}
\hline Patient complaints & Median perceived social pressure & $(\%)$ of Scores $>3^{+}$ \\
\hline Adult with cough & 3 & 28 \\
Adult with fever & 3 & 26 \\
Adult with diarrhea & 4 & 51 \\
Child with cough & 3 & 36.5 \\
Child with fever & 3 & 24.5 \\
Child with diarrhea & 3 & 30 \\
\hline
\end{tabular}

+ Reported as 'often' or 'always' (maximum score = 5) 